\title{
General Purpose Biquads Optimized for Dynamic Range and Low Noise
}

\author{
Dražen Jurišić and Neven Mijat \\ Dept. of Electronic Systems and Information Processing \\ University of Zagreb \\ Unska 3, HR-10 000 Zagreb, Croatia \\ drazen.jurisic@fer.hr; neven.mijat@fer.hr
}

\begin{abstract}
In this paper two general-purpose (GP) biquadratic filters are analyzed and optimized. The design equations for both filters are given, and the methods used to optimize the dynamic range and reduce the noise are described. On one example of the $2^{\text {nd }}$-order Butterworth filter, PSpice analyses were carried out, and a comparison of noise, referred to the input, is given. The dynamic range is optimized such as to have the same signal level at each amplifier output. Both optimization procedures are highly effective and can be applied to the similar filter design problems.
\end{abstract}

\section{INTRODUCTION}

Nowadays, analog active-RC filters (e.g. in [1]) in the VLSI implementation are very often realized by the use of integrators. Because of their easy implementation, a lot of well-known filter topologies tend to be converted into circuits having integrators and appropriate connections between them. Very effective tool used in the analysis and design of such filters is a signal-flow-graph (SFG) representation [2][3]. It enables a straightforward analysis, synthesis and, finally, an optimisation procedure. In what follows, using SFGs, we present two well-known multiamplifier filter biquads: GP sections. We apply the dynamic range optimisation using SFGs method in [4], and we investigate which design procedure provides us with a circuit having optimal thermal (Johnson's) noise performance.

\section{DiRECT REALIZATIONS OF GP SECtions}

We present the well-known realization of biquadratic sections in direct form. Starting from general form of a $2^{\text {nd }}$ order transfer function to build the corresponding SFG, we realize the filter circuit using integrators [5]. Consider the biquadratic transfer function given by:

$$
T(s)=K \frac{b_{2} s^{2}+b_{1} \cdot s+b_{0}}{s^{2}+a_{1} \cdot s+a_{0}} .
$$

Dividing numerator and denominator by $s^{2}$ we obtain:

$$
T(s)=K \frac{b_{2}+b_{1} \frac{1}{s}+b_{0}\left(\frac{1}{s}\right)^{2}}{1+a_{1} \frac{1}{s}+a_{0}\left(\frac{1}{s}\right)^{2}} .
$$

\author{
Ivica Mihalić \\ Siemens \\ Heinzelova 70a, HR-10 000 Zagreb, Croatia \\ ivica.mihalic@siemens.com
}

We can readily calculate a transfer function $T$ between any two nodes in SFG by application of Mason's multipath (general) reduction rule, which is given by:

$$
T=\frac{1}{\Delta} \sum_{k} P_{k} \Delta_{k}
$$

where $\Delta$ is the graph determinant of the form $\Delta=1$-(sum of all loops taken one at a time) $+($ sum of products of all nontouching loops taken two at a time)-(sum of all nontouching loops taken three at a time $)+\ldots . P_{k}$ is the gain of the $k^{\text {th }}$ forward path, and $\Delta_{k}$ is the part of the graph determinant which contains only loops that have no common nodes with the path $P_{k}[2][3]$. Now we can make the following assumptions: (i) every forward path $P_{k}$ touches every loop $L_{i}$ therefore all $\Delta_{k}=1 ;$ (ii) all loops touch each other thus in the graph determinant $\Delta$ all loop products are equal to zero, i.e. $\sum L_{i} L_{j}=0$, $\sum L_{i} L_{j} L_{k^{\prime}}=0$, etc. Thus, we can rewrite (3) in simple form and equate it to (2). We obtain:

$$
T(s)=K \frac{b_{2}+b_{1} \frac{1}{s}+b_{0}\left(\frac{1}{s}\right)^{2}}{1+a_{1} \frac{1}{s}+a_{0}\left(\frac{1}{s}\right)^{2}}=\frac{\sum P_{k}}{1-\sum L_{i}} .
$$

Equality in (4) permits the desired transfer function $T(s)$ given by (2) to be directly realised by the two SFGs that are presented in Fig. 1 in that each coefficient $a_{i}$ and $b_{i}$ $(i=0,1,2)$ appears as a transmission value (see [5]).

Two SFGs in Fig. 1(a) and (b) realize the identical transfer function $V_{\text {out }} / V_{i n}$. It can be seen from (3) for the two SFGs, that the forward paths and loops, as well as topological relations between them, should be the same. We refer to the two graphs, having the above relationship, as transposed. Recall, that the rules by which we can construct the transposed SFG are: $(i)$ change the direction of every branch in the SFG, while keeping the branch transmittances; (ii) make a mirror of the graph; (iii) exchange input and output nodes [2][3].

There are two 3-amplifier realization of $2^{\text {nd }}$-order filter circuits given in [1], i.e. GP-1 and GP-2 sections, based on direct form I and II in Fig. 1, respectively. They provide low-pass (LP), band-pass (BP) and high-pass (HP) outputs [and with an additional opamp also band-rejection (BR) and all-pass (AP) outputs]. 


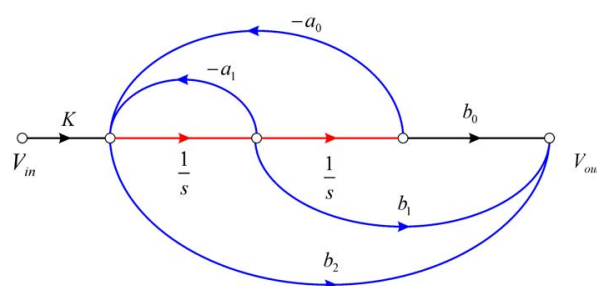

(a)

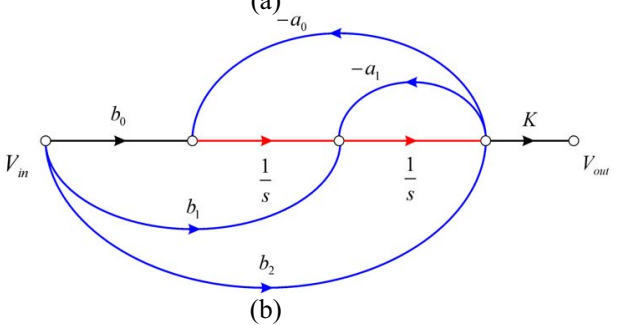

Figure 1. SFG realization of (2). (a) Direct form I. (b) Direct form II

Note that LP, BP and HP outputs correspond to the nodes at opamp outputs. In what follows we demonstrate our optimization method only at the BP filter example, whereas other cases can be treated in the same way.

We demonstrate, as well, that the approach using SFG is very useful in optimization of filter performances.

\section{GP-1 BIQUADRATIC SECTION}

Consider general-purpose realization known as GP-1 biquadratic section shown in Fig. 2, which has two negative integrators with time constants:

$$
\tau_{1}=R_{5} C_{6}, \tau_{2}=R_{7} C_{8} .
$$

The voltage transfer function at the BP output for section in Fig. 2 is given by:

$$
T_{B P}(s)=\frac{V_{\text {out }}}{V_{\text {in }}}=-K_{B P} \frac{\left(\omega_{p} / q_{p}\right) \cdot s}{s^{2}+\left(\omega_{p} / q_{p}\right) \cdot s+\omega_{p}^{2}},
$$

where:

$$
K_{B P}=\frac{R_{2}}{R_{1}}, \omega_{p}^{2}=\frac{R_{4}}{R_{3} \tau_{1} \tau_{2}}, q_{p}=\frac{1+R_{2} / R_{1}}{1+R_{4} / R_{3}} \sqrt{\frac{R_{4} \tau_{1}}{R_{3} \tau_{2}}} .
$$

We represent the circuit in Fig. 2 by a SFG in Fig. 3. Note that (7) permits independent tuning by prescribed noniterative tuning sequence of firstly $\omega_{p}$ by $R_{4}$ and lastly $q_{p}$ by $R_{2}$ [1]. It can be shown that an important quantity that should be minimized in order to minimize the sensitivity to active component variations is the gain-sensitivity product (GSP) of a filter. (The GSP gives a measure of a filter's magnitude sensitivity to the open-loop gain $(A)$ variation of the active component [1].)

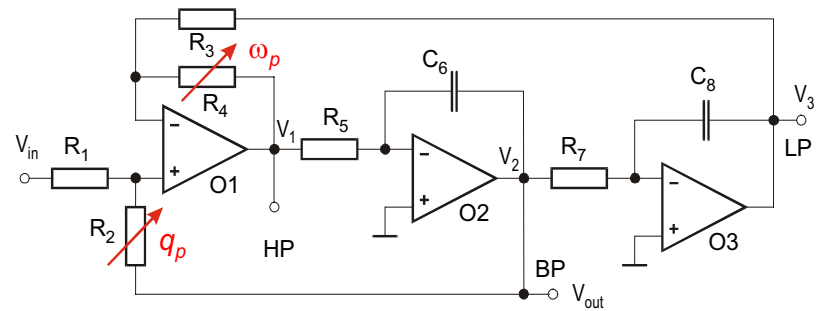

Figure 2. $\quad 2^{\text {nd }}$-order general purpose GP-1 biquad with three opamps

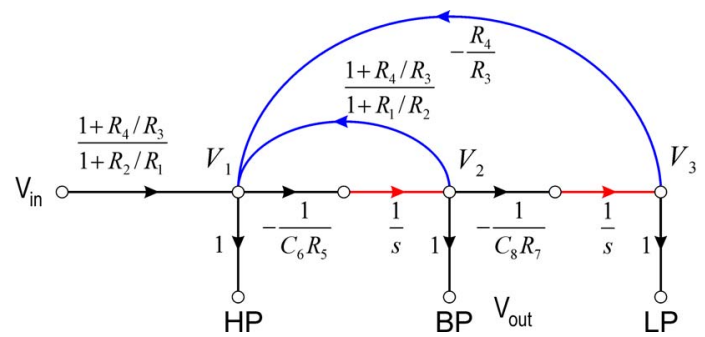

Figure 3. SFG of the GP-1 filter circuit in Fig. 2 showing relationship with component values.

It was shown in [1] that we reduce GSP if we let $\tau_{1}=\tau_{2}$ in (7).

Example: Consider $2^{\text {nd }}$-order Butterworth BP filter having $f_{p}=10 \mathrm{kHz}$ center frequency. To realize this filter we have the pole Q factor, $q_{p}=\omega_{p} / B=0.7071$.

We usually start the design with the normalized center frequency $\omega_{p}=1$ and obtain the normalized component values. Then we denormalize elements to the frequency $\omega_{p}=2 \pi f_{p}$, and to the resistance $R_{0}=\left(\omega_{p} C_{0}\right)^{-1}$, where $f_{p}$ and $C_{0}$ are the desired center frequency and the capacitance. Final resistance and capacitance values follow from:

$$
R_{\text {denorm }}=R_{0} \cdot R_{\text {norm }} ; C_{\text {denorm }}=C_{0} \cdot C_{\text {norm }} .
$$

The step-by-step design procedure for the filter in Fig. 2 . is given in [1], and it provides min-GSP filter:

i) calculate $R_{0}=\left(\omega_{p} C\right)^{-1}$, and choose $R_{d}$; where $R_{0}$ is the optimal resistor for low GSP, and $R_{d}$ is discrete resistor value, which should be close to $R_{0}$;

ii) choose $R_{1}=R_{3}=R_{5}=R_{7}=R_{d}$; and choose $C_{6}=C_{8}=C_{0}$; in doing so we actually choose $\tau_{1}=\tau_{2}$ [see (5)];

iii) finally calculate:

$$
R_{4}=R_{d}\left(R_{d} / R_{0}\right)^{2} ; R_{2}=R_{d}\left(q_{p} \frac{1+R_{4} / R_{d}}{\sqrt{R_{4} / R_{d}}}-1\right) ; K_{B P}=\frac{R_{2}}{R_{1}} \text {. }
$$

The equations for LP and HP gains, $K_{L P}$ and $K_{H P}$ are also given in [1].

After some calculation we obtain $C_{0}=100 \mathrm{pF}$, $R_{0}=159.16 \mathrm{k} \Omega, K_{B P}=0.41421$, and the remaining values that are listed in line 2 in Table I. The corresponding magnitudes of the transfer function characteristics are shown in Fig. 4. Note that the frequency response in Fig. 4 at the output node "BP" within the circuit of Fig. 2 is approx. 1.4142 times lower than that at the output nodes "LP" and "HP". It can happen, as well, that some of the nodes have a peaking above $0 \mathrm{~dB}$, thus signal overdrive and distortion may ensue at these nodes.

TABLE I. COMPONENT VALUES FOR GP-1 FILTER $(R \mathrm{~s}$ in $[\mathrm{k} \Omega], C \mathrm{~s}$ in $[\mathrm{pF}])$

\begin{tabular}{|c|c|c|c|c|c|c|c|c|}
\hline No. & Filter & $\mathbf{R}_{\mathbf{1}}=\mathbf{R}_{\mathbf{3}}=\mathbf{R}_{\mathbf{4}}$ & $\mathbf{R}_{\mathbf{2}}$ & $\mathbf{R}_{\mathbf{5}}$ & $\mathbf{C}_{\mathbf{6}}$ & $\mathbf{R}_{\mathbf{7}}$ & $\mathbf{C}_{\mathbf{8}}$ & $\mathbf{R}_{\text {TOT }}$ \\
\hline 1) & $0.7 \tau_{1}=\tau_{2}$ & 159.16 & 32.16 & 187.24 & 100 & 135.28 & 100 & 832.16 \\
\hline $\mathbf{2 )}$ & $\tau_{1}=\tau_{2}$ & 159.16 & 65.92 & 159.16 & 100 & 159.16 & 100 & 861.72 \\
\hline $\mathbf{3 )}$ & $2 \tau_{1}=\tau_{2}$ & 159.16 & 159.16 & 112.54 & 100 & 225.08 & 100 & 974.26 \\
\hline $\mathbf{4 )}$ & $4 \tau_{1}=\tau_{2}$ & 159.16 & 291.0 & 79.58 & 100 & 318.3 & 100 & 1166.4 \\
\hline
\end{tabular}




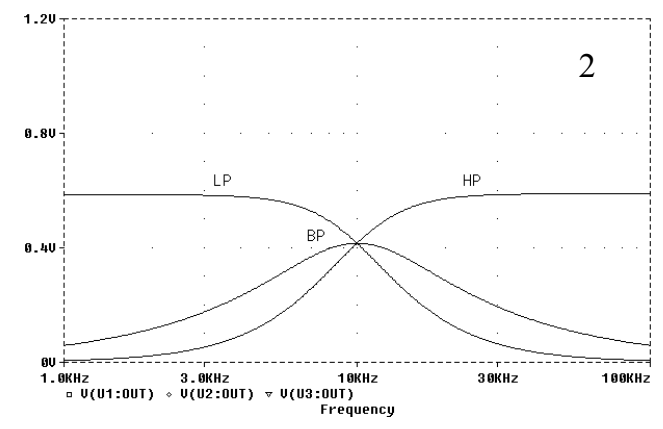

Figure 4. Magnitudes of all outputs of the GP-1 filter no. 2 in Table I.

One criterion that is useful to guarantee max. dynamic range is to specify that the maximum signal level at any node within the circuit should at no time exceed the signal level at the input or output. On the other hand if some of the nodes have much lower signal level than those at the other nodes, it also deteriorates the dynamic properties of the filter. Thus, for input signal equal to $1 \mathrm{~V}$ the largest signal within the circuit should everywhere in the circuit be equal to $1 \mathrm{~V}$.

A simple way of signal level scaling within a circuit is to consider the equivalent SFG as given in Fig. 3. Now, to change (i.e., increase or decrease by an amount $\lambda_{1}$ ) the voltage level inside any cutset of the graph, we must multiply every incoming branch by the factor $\lambda_{1}$ and divide every outgoing branch by the same amount. (A cutset is a minimal set of branches which, when removed, will separate the graph into exactly two parts: subgraphs-a subgraph can be a single node, as well). Thus, if the signal level at node BP is to be changed by a factor $\lambda_{1}$, we obtain the new SFG given in Fig. 5 with subgraph (containing node $V_{2}$ ) surrounded by a dashed circle. Here all incoming signal paths to the subgraph, have been multiplied by $\lambda_{1}$, while all outgoing paths have been divided by $\lambda_{1}$. To do so we must multiply or divide one multiplicative component in the corresponding transmission quantity. In Fig. 5 we do this by dividing $R_{5}$ and multiplying $R_{7}$ by $\lambda_{1}$, and calculating new ratio $R_{2} / R_{1}$ (as a function of $\lambda_{1}$ ). In our example we have found the scaling factor $\lambda_{1}=1.4142$, numerically, observing magnitudes in Fig. 4, that are calculated using PSpice.

Finally, we obtain optimized component values of the filter no. 3 in Table I, for which: (i) $\tau_{1}=\tau_{2} / 2$; (ii) the amplitudes at all filter (opamp) outputs (see Fig. 6), all reach the maximum value of $1 \mathrm{~V}$. Magnitudes of (not optimized for dynamic range) filters no. 1 and 4 are not presented.

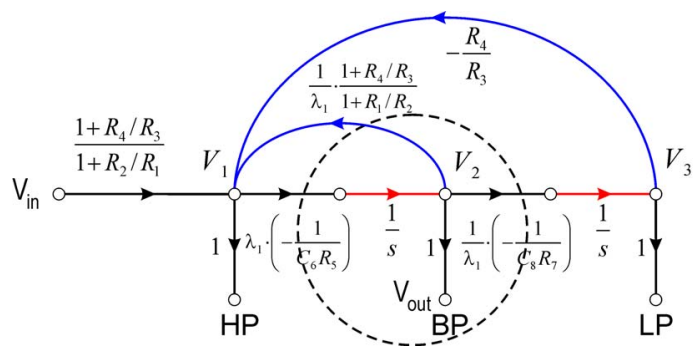

Figure 5. SFG of Fig. 3 with output BP scaled by $\lambda_{1}$

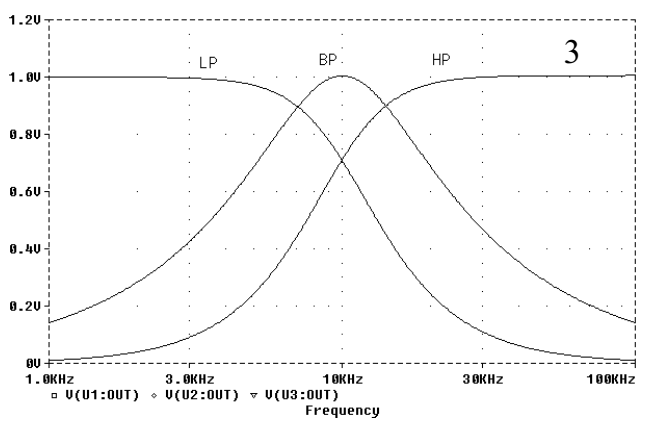

Figure 6. Magnitudes of all outputs of the GP-1 filter no. 3 in Table I

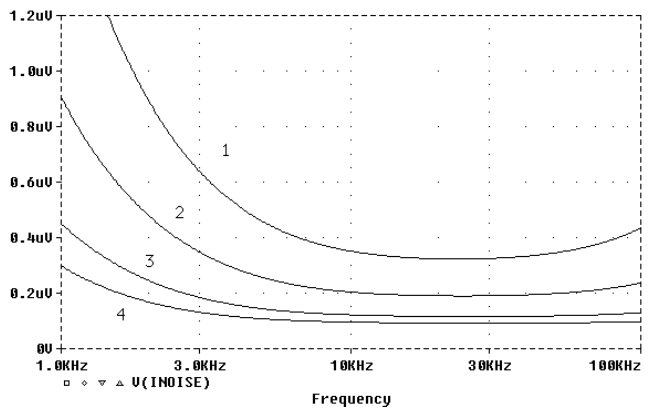

Figure 7. Input noise of the BP output of the GP-1 filters in Table I.

Noise referred to the input was calculated for the examples in Table I using PSpice and shown in Fig. 7. In spite of ascending total resistance level (see $R_{\text {TOт }}$ column in Table I), the noise is getting lower for the case when $\tau_{1}$ is getting larger than $\tau_{2}$ and it is minimum for the circuit no. 4. It is recommended, when applying the GP filters, they should be designed with the maximum total permissible capacitance values (from an IC-technology point of view) in order to minimize the thermal noise.

Consequently the most promising circuit is no. 3, which has both lower noise and optimized dynamic range, whereas the circuit no. 2 has minimum GSP. Thus, which way to choose is one of typical trade-offs in filter design.

\section{GP-2 Biquadratic Section}

Consider general-purpose realization GP-2 biquadratic section shown in Fig. 8, which has integrators with $\tau \mathrm{s}$ :

$$
\tau_{1}=R_{2} C_{3}, \tau_{2}=R_{5} C_{6} .
$$

The voltage transfer function at the BP output for this section has the form (6), with:

$$
K_{B P}=\frac{R_{4}}{R_{1}}, \omega_{p}^{2}=\frac{R_{8}}{R_{7} \tau_{1} \tau_{2}}, q_{p}=R_{4} C_{3} \omega_{p},
$$

We can represent the circuit in Fig. 8 by a SFG in Fig. 9.

Step-by-step design procedure for the GP-2 filter in Fig. 8. is given in [1], and it provides minimum-gainsensitivity-product (min-GSP) filter circuit:

i) calculate $R_{0}=\left(\omega_{p} C\right)^{-1}$, and accordingly choose $R_{d} \approx R_{0}$;

ii) choose $R_{2}=R_{7}=R_{8}=R_{d}$; and choose $C_{3}=C_{6}=C_{0}\left(\tau_{1}=\tau_{2}\right)$;

iii) finally calculate: 


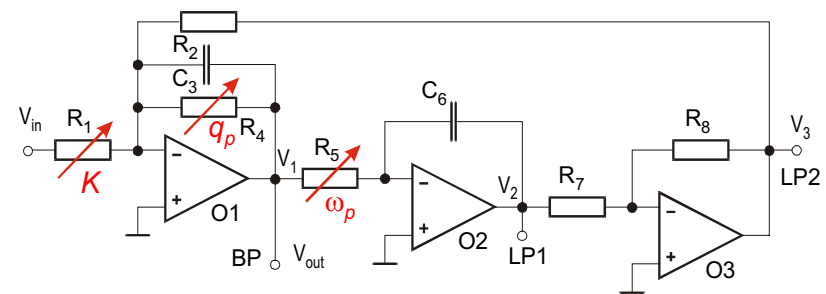

Figure 8. $2^{\text {nd }}$-order general purpose GP-2 biquad.

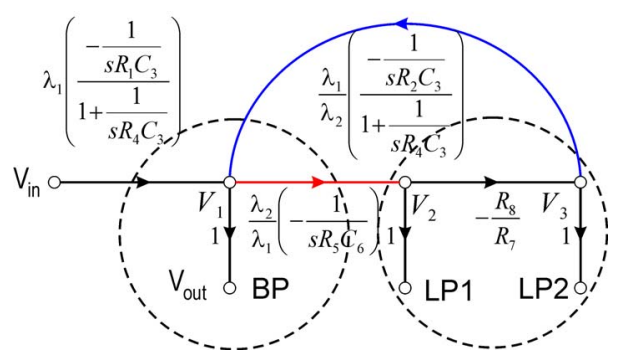

Figure 9. SFG of the circuit in Fig. 8 with component values. Outputs BP and LP are scaled by $\lambda_{1}$ and $\lambda_{2}$, respectively.

$$
R_{5}=R_{d}\left(R_{0} / R_{d}\right)^{2}: R_{2}=\frac{q_{p}}{\omega_{p} C_{3}} ; R_{1}=\frac{R_{4}}{K_{B P}} .
$$

After calculation with $\tau_{1}=\tau_{2}$ we obtain filter component values listed in line 1 in Table II. The corresponding magnitude is shown in Fig. 10(a), from which we see that the frequency response at the node "BP" within the circuit is approximately 1.4142 times lower than that at the "LP".

TABLE II. DENORMALIZED COMPONENT VALUES FOR GP-2 FILTER.

\begin{tabular}{|c|c|c|c|c|c|c|c|c|}
\hline No. & Filter & $\mathbf{R}_{\mathbf{1}}$ & $\mathbf{R}_{\mathbf{2}}$ & $\mathbf{C}_{\mathbf{3}}$ & $\mathbf{R}_{\mathbf{4}}$ & $\mathbf{R}_{\mathbf{5}}$ & $\mathbf{C}_{\mathbf{6}}$ & $\mathbf{R}_{\mathbf{7}}=\mathbf{R}_{\mathbf{8}}$ \\
\hline $\mathbf{1 .}$ & $\tau_{1}=\tau_{2}$ & 225.08 & 159.16 & 100 & 112.54 & 159.16 & 100 & 159.16 \\
\hline $\mathbf{2 .}$ & $2 \tau_{1}=\tau_{2}$ & 112.54 & 112.54 & 100 & 112.54 & 225.08 & 100 & 159.16 \\
\hline
\end{tabular}

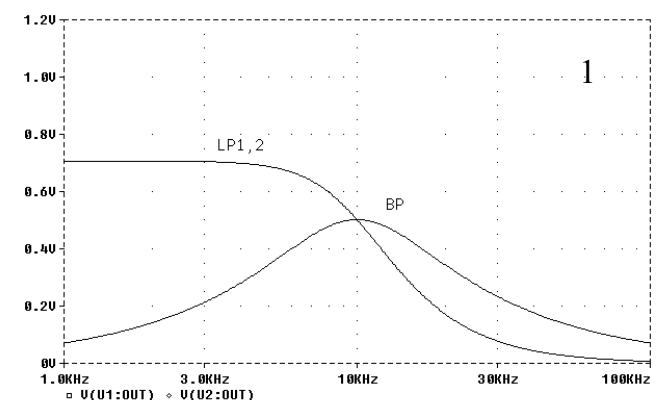

(a) Frequency

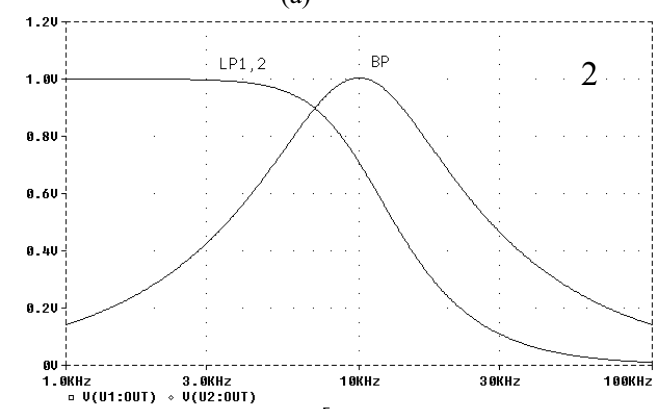

(b)

Figure 10. Magnitudes of all outputs of the GP-2 filters in Table II. (a) Filter eg. no. 1. (b) Dynamic-range-optimized filter eg. no. 2.

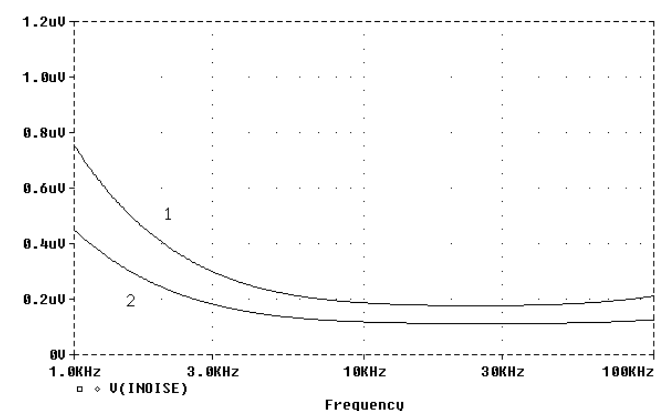

Figure 11. Input noise of the BP output of the GP-2 filters in Table II.

To maximize dynamic range of the filter circuit no. 1 in Table II we have to scale its voltage levels at nodes $V_{1}$ and $V_{2}\left(V_{2}=V_{3}\right)$ by factors $\lambda_{1}=1.4142$ and $\lambda_{2}=2$, respectively, as shown in SFG in Fig. 9. To optimize dynamic range we must scale one component in the corresponding SFG branch. In Fig. 9 we do this by dividing $R_{1}$ by $\lambda_{1}$, multiplying $R_{5}$ by $\lambda_{1} / \lambda_{2}$, and finally multiplying $R_{2}$ by the ratio $\lambda_{2} / \lambda_{1}$. We obtain component values of the filter no. 2 in Table II and the transfer function magnitudes shown in Fig. 10(b). Notice that the latter filter is well optimized for the dynamic range.

PSpice runs of the input noise (reduced from the BP output) emanating from the multiamplifier biquad in Fig. 8 are shown in Fig. 11 for the two filter examples in Table II. It can be seen again, that, luckily, the filter no. 2 optimized for dynamic range has lower noise than the filter no. 1, which was designed for min. GSP according to [1]. As already stated above, it is the trade off in the filter design. If we compare two optimized filter examples no. 3 in Table I (i.e. GP-1) and no. 2 in Table II (i.e. GP2), regarding input noise in Figs. 7 and 11, we can see that both GP-1 and GP-2 sections have equal noise performance.

\section{CONCLUSION}

In this paper we presented design equations for the two commonly used GP filter types and a method of optimizing theirs dynamic range. PSpice analyses of the frequency response, and simulations of thermal noise, referred to the input, were carried out. It is recommended, that the GP filters be designed with minimized noise, and optimized dynamic range by signal scaling at appropriate circuit nodes. Both procedures are highly effective and may well be used in all other similar filter designs. It was demonstrated that both GP-1 and GP-2 biquads have identical performance regarding noise.

\section{REFERENCES}

[1] G. S. Moschytz and P. Horn, Active Filter Design Handbook. Chichester, U.K.: Wiley 1981.

[2] S. J. Mason, "Feedback theory-Some properties of signal flow graphs," Proc. IRE, vol. 41, no. 9, pp. 1144-1156, Sept. 1953.

[3] S. J. Mason, "Feedback theory-Further properties of signal flow graphs,” Proc. IRE, vol. 44, no. 7, pp. 920-926, July 1956.

[4] D. J. Perry, "Scaling Transformation of Multiple-Feedback Filters”, Proc. IEE, vol.128, pp.176-179, Aug. 1981.

[5] G. S. Moschytz, "An introduction to signal-flow graph theory," in course Analog signal processing and related bipolar and CMOS circuit design, CEI-Europe, Advanced technology education, Sweden. 